CERN-PH-TH-2013-165

FERMILAB-FN-0963-CD-T

\title{
The Matrix Element Method: Past, Present, and Future
}

\author{
James S. Gainer, ${ }^{1}$ Joseph Lykken, ${ }^{2}$ Konstantin T. Matchev, ${ }^{1}$ Stephen Mrenna, ${ }^{3}$ and Myeonghun Park ${ }^{4}$ \\ ${ }^{1}$ Physics Department, University of Florida, Gainesville, FL 32611, USA \\ ${ }^{2}$ Theoretical Physics Department, Fermilab, Batavia, IL 60510, USA \\ ${ }^{3}$ SSE Group, Computing Division, Fermilab, Batavia, IL 60510, USA \\ ${ }^{4}$ Theory Division, Physics Department, CERN, CH-1211 Geneva 23, Switzerland
}

(Dated: July 26, 2013)

\begin{abstract}
The increasing use of multivariate methods, and in particular the Matrix Element Method (MEM), represents a revolution in experimental particle physics. With continued exponential growth in computing capabilities, the use of sophisticated multivariate methods- already common- will soon become ubiquitous and ultimately almost compulsory. While the existence of sophisticated algorithms for disentangling signal and background might naively suggest a diminished role for theorists, the use of the MEM, with its inherent connection to the calculation of differential cross sections will benefit from collaboration between theorists and experimentalists. In this white paper, we will briefly describe the MEM and some of its recent uses, note some current issues and potential resolutions, and speculate about exciting future opportunities.
\end{abstract}

Introduction- Multivariate methods [1] are widely used in experimental particle physics; popular examples include boosted decision trees (BDT) and neural nets, in addition to the Matrix Element Method (MEM) [2], which will be the subject of our discussion here. In the MEM, the likelihood for a given event, with measured momenta $\mathbf{p}_{i}^{\text {vis }}$ in some underlying model with parameters $\alpha$ is given by

$$
\begin{aligned}
\mathcal{P}\left(\mathbf{p}_{i}^{\mathrm{vis}} \mid \alpha\right) & =\frac{1}{\sigma(\alpha)} \sum_{k, l} \int d x_{1} d x_{2} \frac{f_{k}\left(x_{1}\right) f_{l}\left(x_{2}\right)}{2 s x_{1} x_{2}} \\
& \times\left[\prod_{j \in \text { inv. }} \int \frac{d^{3} p_{j}}{(2 \pi)^{3} 2 E_{j}}\right]\left|\mathcal{M}_{k l}\left(p_{i}^{\mathrm{vis}}, p_{j} ; \alpha\right)\right|^{2},
\end{aligned}
$$

where $f_{k}$ and $f_{l}$ are parton distribution functions, $\mathcal{M}_{k l}$ is the theoretical matrix element, and $\left.\sigma_{(} \alpha\right)$ is the (total) cross section after cuts and efficiencies. If the process involves invisible particles, such as neutrinos or neutralinos, then their momenta, $p_{j}$, must be integrated over the appropriate phase space.

Transfer functions parameterizing the detector resolution, should also be included and integrated over, as the matrix element is a function of the actual, rather than the observed, particle momenta. In the limit where all the quantities and functions in Eq. 1 1 are known with perfect accuracy, the quantity calculated in this manner is the likelihood, and hence by the Neyman-Pearson Lemma is an optimal test statistic [3]. Hence, if it can be implemented, the MEM should be the most sensitive analysis possible - we will note some caveats later in this paper.

Past and Present- The MEM has been used in studies of top properties at the Tevatron [4], as well as in B physics [5]. A notable recent application has been in the study of $H \rightarrow Z Z^{*} \rightarrow 4 \ell$ [6 10], where the MEM, in the form of the "MELA" approach [8], was used in the Higgs discovery by CMS 11. Currently CMS uses MELA together with MEKD [9, 12], another MEM package in continuing studies of the properties of the Higgs. ATLAS is using a BDT for similar studies [13].

The MEM has also been suggested for studying the Higgs in other channels [14, 15] and for BSM energy frontier physics [16]. A dedicated package, MadWeight, has been developed for MEM studies [17].

Near Future- A practical challenge associated with the use of the MEM is that the likelihood calculated is only an approximation of the true likelihood. This situation arises because of (i) finite detector resolution (ii) higher order corrections (iii) neglected information. Therefore, rather than taking e.g. the likelihood ratio calculated from the MEM directly to have a given statistical significance, it may still be necessary for an experiment to calculate the statistical significance of a given result using e.g. pseudoexperiments, which naively can be very expensive from the standpoint of computer time.

We note that the detector response to an event is generally independent of the underlying parameters of the model which described the hard process (e.g. masses or couplings of virtual particles produced in the collision). Thus one can often simply re-weight the events already generated in a given pseudo-experiment to calculate the likelihood at any other point in the parameter space [10]. This procedure eliminates the need to generate a separate event sample for each point in parameter space and thus significantly speeds up the analysis. An analogous procedure could be employed for template-based analyses.

Future- Moore's Law [18] predicts that the vast increases in computing power that have characterized the 
past decades will continue. Even today, technological advances such as the use of graphics processing units [19] may significantly increase the computing resources available for analyses. If we assume that this trend will continue, then ultimately we will have the computing power to perform analyses that would be wildly impractical at the present. We therefore speculate about future developments involving the MEM, without regard to (current) computational limitations.

- NLO/ Parton Showers- The extension of the MEM to take into account additional radiation and/or other NLO corrections has already been considered [7, 15, 20]. Work has also gone into extending the MEM to include parton showers 21]. Such approaches, potentially extended beyond NLO, will leverage the more complete higher order calculations of the future.

- Jet Substructure- As outlined in Eq. 1 the MEM is only using the four momentum of a jet. However, other properties of the jet, e.g. substructure [22] could give additional information about the hard process parton with which a jet should be associated, thereby increasing the sensitivity of the analysis.

- Underlying Event, Hadronization, Etc.- Additional information about hadronization, the underlying event, and other topics could also be used to calculate a more complete likelihood for events in hadron colliders.

- Detector Resolution- Ultimately, instead of using a single transfer function for a detector, a separate transfer function could be utilized for each detector element involved in the reconstruction of an event. Ideally the time dependence of the element response, as well as its correlations with other detector elements would be included.

Conclusions- The future developments of the MEM described above will require heroic efforts of theorists and experimentalists ${ }^{1}$. The result will be a significant increase in the ability of future colliders to perform precision measurements and searches.

Acknowledgments- MP is supported by the CERNKorea fellowship through the National Research Foundation of Korea. Work supported in part by U.S. Department of Energy Grants DE-FG02-97ER41029. Fermilab is operated by the Fermi Research Alliance under contract DE-AC02-07CH11359 with the U.S. Department of Energy.

\footnotetext{
1 An annual workshop to discuss MEM developments was initiated this year 23]
}

[1] P. C. Bhat, "Multivariate Analysis Methods in Particle Physics," Ann. Rev. Nucl. Part. Sci. 61, 281 (2011).

[2] K. Kondo, J. Phys. Soc. Jap. 57, 4126 (1988) and 60, 836 (1991); R. H. Dalitz and G. R. Goldstein, Phys. Rev. D 45, 1531 (1992).

[3] J. Neyman and E. S. Pearson, Phil. Trans. R. Soc. Lond. A 231 no. 694-706, 289-337 (1933).

[4] B. Abbott et al. [DØ Collaboration], Phys. Rev. D 60, 052001 (1999); J. C. Estrada Vigil, FERMILABTHESIS-2001-07; M. F. Canelli, UMI-31-14921; V. M. Abazov et al. [D $\varnothing$ Collaboration], Nature 429, 638 (2004); A. Abulencia et al. [CDF Collaboration], Phys. Rev. D 74, 032009 (2006); A. Abulencia et al. [CDF Collaboration], Phys. Rev. D 75, 031105 (2007); V. M. Abazov et al. [DØ Collaboration], Phys. Rev. D 78, 012005 (2008); T. Aaltonen et al. [CDF Collaboration], Phys. Rev. Lett. 101, 252001 (2008); F. Fiedler, A. Grohsjean, P. Haefner and P. Schieferdecker, Nucl. Instrum. Meth. A 624, 203 (2010); F. Fiedler, A. Grohsjean, P. Haefner and P. Schieferdecker, Nucl. Instrum. Meth. A 624, 203 (2010); T. Aaltonen et al. [CDF Collaboration], CDF/PHYS/TOP/PUBLIC/10191 (2010).

[5] I. Dunietz, H. R. Quinn, A. Snyder, W. Toki and H. J. Lipkin, Phys. Rev. D 43, 2193 (1991); G. Kramer and W. F. Palmer, Phys. Rev. D 45, 193 (1992); A. V. Gritsan and J. G. Smith, "Polarization in B Decays," in J. Beringer et al. [Particle Data Group Collaboration], Phys. Rev. D 86, 010001 (2012).

[6] A. De Rujula, J. Lykken, M. Pierini, C. Rogan and M. Spiropulu, Phys. Rev. D 82, 013003 (2010) arXiv:1001.5300 [hep-ph]]; J. S. Gainer, K. Kumar, I. Low and R. Vega-Morales, JHEP 1111, 027 (2011) arXiv:1108.2274 [hep-ph]]; D. Stolarski and R. Vega-Morales, Phys. Rev. D 86, 117504 (2012) arXiv:1208.4840 [hep-ph]]; T. Modak, D. Sahoo, R. Sinha and H. -Y. Cheng, arXiv:1301.5404 [hep-ph].

[7] J. M. Campbell, W. T. Giele and C. Williams, arXiv:1205.3434 [hep-ph]; J. M. Campbell, W. T. Giele and C. Williams, JHEP 1211, 043 (2012) arXiv:1204.4424 [hep-ph]].

[8] Y. Gao, A. V. Gritsan, Z. Guo, K. Melnikov, M. Schulze and N. V. Tran, Phys. Rev. D 81, 075022 (2010) arXiv:1001.3396 [hep-ph]]; S. Bolognesi, Y. Gao, A. V. Gritsan, K. Melnikov, M. Schulze, N. V. Tran and A. Whitbeck, Phys. Rev. D 86, 095031 (2012) arXiv:1208.4018 [hep-ph]].

[9] P. Avery, D. Bourilkov, M. Chen, T. Cheng, A. Drozdetskiy, J. S. Gainer, A. Korytov and K. T. Matchev et al., Phys. Rev. D 87, 055006 (2013) arXiv:1210.0896 [hep$\mathrm{ph}$.

[10] J. S. Gainer, J. Lykken, K. T. Matchev, S. Mrenna and M. Park, arXiv:1304.4936 [hep-ph].

[11] S. Chatrchyan et al. [CMS Collaboration], JHEP 1204, 036 (2012) arXiv:1202.1416 [hep-ex]]; S. Chatrchyan et al. [CMS Collaboration], Phys. Lett. B 716, 30 (2012) arXiv:1207.7235 [hep-ex]].

[12] S. Chatrchyan et al. [CMS Collaboration], Phys. Rev. Lett. 110, 081803 (2013) arXiv:1212.6639 [hep-ex]]; [CMS Collaboration], CMS-PAS-HIG-12-041; [CMS Collaboration], CMS-PAS-HIG-13-002. 
[13] G. Aad et al. [ATLAS Collaboration], arXiv:1307.1432 [hep-ex].

[14] K. Cranmer and T. Plehn, Eur. Phys. J. C 51, 415 (2007) hep-ph/0605268; S.-C. Hsu et al. [CDF Collaboration], CDF note 8774 (2007); T. Aaltonen et al. [CDF Collaboration], Phys. Rev. D 80, 071101 (2009); J. Therhaag, Diplom thesis, University of Bonn, BONNIB-2009-03 (2009); J. S. Gainer, W.-Y. Keung, I. Low and P. Schwaller, Phys. Rev. D 86, 033010 (2012); J. R. Andersen, C. Englert and M. Spannowsky, arXiv:1211.3011 [hep-ph]; J. R. Andersen, C. Englert and M. Spannowsky, Phys. Rev. D 87, 015019 (2013) arXiv:1211.3011 [hepph]]; A. Freitas and J. S. Gainer, arXiv:1212.3598 [hepph]; P. Artoisenet, P. de Aquino, F. Maltoni and O. Mattelaer, arXiv:1304.6414 [hep-ph]; P. Artoisenet, P. de Aquino, F. Demartin, R. Frederix, S. Frixione, F. Maltoni, M. K. Mandal and P. Mathews et al., arXiv:1306.6464 [hep-ph].

[15] J. M. Campbell, R. K. Ellis, W. T. Giele and C. Williams, Phys. Rev. D 87, 073005 (2013) arXiv:1301.7086 [hep- $\mathrm{ph}]$.

[16] J. Alwall, A. Freitas and O. Mattelaer, AIP Conf. Proc. 1200, 442 (2010); C.-Y. Chen and A. Freitas, JHEP 1102, 002 (2011); O. Gedalia, G. Isidori, F. Maltoni, G. Perez, M. Selvaggi and Y. Soreq, arXiv:1212.4611 [hep-ph].

[17] P. Artoisenet, V. Lemaître, F. Maltoni and O. Mattelaer, JHEP 1012, 068 (2010).

[18] G. E. Moore, Electronics, 38, Number 8, 4 (1965).

[19] https://en.wikipedia.org/wiki/Graphics_processing_unit

[20] J. Alwall, A. Freitas and O. Mattelaer, Phys. Rev. D 83, 074010 (2011).

[21] D. E. Soper and M. Spannowsky, Phys. Rev. D 84, 074002 (2011) arXiv:1102.3480 [hep-ph]]; D. E. Soper and M. Spannowsky, arXiv:1211.3140 [hep-ph].

[22] J. Shelton, "TASI Lectures on Jet Substructure," arXiv:1302.0260 [hep-ph].

[23] https://agenda.irmp.ucl.ac.be/

conferenceDisplay.py? conf $\mathrm{Id}=1502$ 